\title{
Unilateral Global Bifurcation for Fourth-Order Problems and Its Applications
}

\author{
Wenguo Shen \\ Department of Basic Courses, Lanzhou Institute of Technology, Lanzhou 730050, China \\ Correspondence should be addressed to Wenguo Shen; shenwg369@163.com
}

Received 10 January 2016; Accepted 12 June 2016

Academic Editor: Cengiz Çinar

Copyright (c) 2016 Wenguo Shen. This is an open access article distributed under the Creative Commons Attribution License, which permits unrestricted use, distribution, and reproduction in any medium, provided the original work is properly cited.

\begin{abstract}
We will establish unilateral global bifurcation result for a class of fourth-order problems. Under some natural hypotheses on perturbation function, we show that $\left(\lambda_{k}, 0\right)$ is a bifurcation point of the above problems and there are two distinct unbounded continua, $C_{k}^{+}$and $C_{k}^{-}$, consisting of the bifurcation branch $C_{k}$ from $\left(\mu_{k}, 0\right)$, where $\mu_{k}$ is the $k$ th eigenvalue of the linear problem corresponding to the above problems. As the applications of the above result, we study the existence of nodal solutions for the following problems: $x^{\prime \prime \prime \prime}+k x^{\prime \prime}+l x=r h(t) f(x), 0<t<1, x(0)=x(1)=x^{\prime}(0)=x^{\prime}(1)=0$, where $r \in \mathbb{R}$ is a parameter and $k, l$ are given constants; $h(t) \in C([0,1],[0, \infty))$ with $h(t) \neq \equiv$ on any subinterval of $[0,1]$; and $f: \mathbb{R} \rightarrow \mathbb{R}$ is continuous with $s f(s)>0$ for $s \neq 0$. We give the intervals for the parameter $r \neq 0$ which ensure the existence of nodal solutions for the above fourth-order Dirichlet problems if $f_{0} \in[0, \infty]$ or $f_{\infty} \in[0, \infty]$, where $f_{0}=\lim _{|s| \rightarrow 0} f(s) / s$ and $f_{\infty}=\lim _{|s| \rightarrow+\infty} f(s) / s$. We use unilateral global bifurcation techniques and the approximation of connected components to prove our main results.
\end{abstract}

\section{Introduction}

The deformations of an elastic beam in equilibrium state with fixed both endpoints can be described by the fourth-order boundary value problem

$$
\begin{aligned}
x^{\prime \prime \prime \prime}+k x^{\prime \prime}+l x & =r h(t) f(x), \quad 0<t<1, \\
x(0) & =x(1)=x^{\prime}(0)=x^{\prime}(1)=0,
\end{aligned}
$$

where $r \in \mathbb{R}$ is a parameter, $k, l$ are given constants, and $f: \mathbb{R} \rightarrow \mathbb{R}$ is continuous. When $k=l=0$, since problem (1) cannot transform into a system of second-order equation, the treatment method of second-order system does not apply to problem (1). Thus, there exists some difficulty studying problem (1) even in the case of $k=l=0$.

In recent years, there has been considerable interest in the above BVP (1) mainly because of their interesting applications. For example, Agarwal and Chow [1] $(k=l=0)$ first investigated the existence of the solutions of problem (1) by contraction mapping and iterative methods. Subsequently, when $k=l=0$, by fixed point theory on cones, Ma et al. $[2,3]$, Yao [4, 5], Zhai et al. [6], and Webb et al. [7] studied the existence of positive solutions of problem (1).
On the other hand, by applying the bifurcation techniques of Rabinowitz [8,9], Gupta and Mawhin [10], Lazer and McKenna [11], Liu and O'Regan [12], and Ma et al. [13-15] studied the existence of nodal solutions for the fourth-order BVP where both ends were simply supported, and Rynne [16] investigated the nodal properties of the solutions for a general $2 m$ th-order problem.

Meanwhile, it is well known that the spectrum structure of the linear eigenvalue problems according to (1) plays a key role to study problem (1) by the bifurcation techniques. Kratochvil and Nečas [17] first studied the spectrum of the $p$ biharmonic operator together with $x(0)=x(1)=x^{\prime}(0)=$ $x^{\prime}(1)=0$. Subsequently, Benedikt [18-21] also studied the spectral properties of the corresponding eigenvalue problem of the same problems as [17], and Benedikt [22] studied existence and global bifurcation of solutions for the above problems. When $k=l=0$, by applying the bifurcation techniques, Korman [23] investigated the uniqueness of positive solutions and Rynne [24] studied nodal properties of the solutions for problem (1), respectively. By Elias's theory [25, 26], Xu and Han [27] $(k=0)$, Ma et al. [28] $(k=0, l=l(t))$, and Ma and Gao [29] $\left(x^{\prime \prime \prime \prime}+k x^{\prime \prime}+l x=(p(t) x)^{\prime \prime \prime \prime}-(q(t) x)^{\prime \prime}\right)$ established the spectrum structure of the linear eigenvalue 
problems according to (1) and studied the existence of nodal solutions of problem (1) using bifurcation theory [8]. In 2012, Shen $[30,31]$ established the following spectrum structure by applying disconjugate operator theory $[25,26]$.

Lemma 1 (see $[30,31])$. Let $(A 1)$ and $(A 2)$ hold. The linear eigenvalue problem

$$
\begin{aligned}
x^{\prime \prime \prime \prime}(t)+k x^{\prime \prime}(t)+l x(t) & =\lambda h(t) x, \quad 0<t<1, \\
x(0) & =x(1)=x^{\prime}(0)=x^{\prime}(1)=0
\end{aligned}
$$

has a unique infinite number of positive eigenvalues

$$
0<\lambda_{1}<\lambda_{2}<\cdots<\lambda_{k}<\cdots \rightarrow \infty, \text { as } k \rightarrow \infty \text {. }
$$

Moreover, each eigenvalue is simple. The eigenfunction $\psi_{k}$ corresponding to $\lambda_{k}$ has exactly $k-1$ simple zeros in $(0,1)$. For each $k \in \mathbb{N}$, the algebraic multiplicity of $\lambda_{k}$ is 1 , where $(A 1)$ one of following conditions holds:

(i) $k$, l satisfying $(k, l) \in\{(k, l) \mid k \in(-\infty, 0], l \in(0, \infty)\} \backslash$ $\left\{\left(0, \pi^{4} / 64\right)\right\} \cup\left\{(k, l) \mid k \in\left(-\infty, \pi^{2}\right), l \in(-\infty, 0]\right\}$, are given constants with

$$
\pi^{2}\left(k-\pi^{2}\right)<l \leq \frac{1}{4}\left(k-\frac{\pi^{2}}{4}\right)^{2} .
$$

(ii) $k, l$ satisfying $(k, l) \in\left\{(k, l) \mid k \in\left(0, \pi^{2} / 2\right), l \in\right.$ $(0, \infty)\}$, are given constants with

$$
\frac{1}{4}\left(\pi^{2} k-\frac{\pi^{4}}{4}\right)<l \leq \frac{1}{4} k^{2} .
$$

$(A 2) h(t) \in C([0,1],[0, \infty))$ with $h(t) \quad \equiv \quad 0$ on any subinterval of $[0,1]$.

On the basis of Lemma 1, Shen [30, 31] studied the existence of nodal solutions of problem (1) by applying Rabinowitz's global bifurcation theorem [8].

In 2013, when $k, l$ satisfy $(A 1)$ and $(A 2)$, Shen and $\mathrm{He}$ [32] also studied bifurcation from interval and the existence of positive solutions for problem (1) by applying Rabinowitz's global bifurcation theorem [9].

Now, consider the following operator equation:

$$
u=\lambda B u+H(\lambda, u) \text {, }
$$

where $B$ is a compact linear operator and $H: \mathbb{R} \times E \rightarrow E$ is compact with $H=o(\|u\|)$ at $u=0$ uniformly on bounded $\lambda$ intervals, where $E$ is a real Banach space with the norm $\|\cdot\|$. If the eigenvalue $\mu$ of $B$ has multiplicity 1 ,

$$
\mathcal{S}=\overline{\{(\lambda, u):(\lambda, u) \text { satisfies (6) and } u \neq \equiv 0}^{\mathbb{R} \times E} \text {. }
$$

Dancer [33] has shown that there are two distinct unbounded continua $C_{\mu}^{+}$and $C_{\mu}^{-}$, consisting of the bifurcation branch $C_{\mu}$ of $\mathcal{S}$ emanating from $(\mu, 0)$, which satisfy either that $C_{\mu}^{+}$and $C_{\mu}^{-}$are both unbounded or $C_{\mu}^{+} \cap C_{\mu}^{-} \neq\{(\mu, 0)\}$. This result has been extended to one-dimensional $p$-Laplacian problem by
Dai and Ma [34]. The above results [34] have been improved partially by Dai [35] with nonasymptotic nonlinearity at 0 or $\infty$. Later, Dancer's result [33] has been also extended to the periodic $p$-Laplacian problems by Dai et al. [36]. In 2013, Dai and Han [37] established Dancer-type unilateral global bifurcation results for fourth-order problems of the deformations of an elastic beam in equilibrium state where both ends are simply supported by Dancer [33].

In this paper, based the spectral theory of $[30,31]$, we will establish Dancer-type unilateral global bifurcation results about the continuum of solutions for the following fourthorder eigenvalue problem:

$$
\begin{gathered}
x^{\prime \prime \prime \prime}+k x^{\prime \prime}+l x=\lambda h(t) x+g(t, x, \lambda), \quad 0<t<1, \\
x(0)=x(1)=x^{\prime}(0)=x^{\prime}(1)=0,
\end{gathered}
$$

where $h$ satisfies (A2), and the perturbation function $g$ : $(0,1) \times \mathbb{R}^{2} \rightarrow \mathbb{R}$ is continuous with $g(t, s, 0) \equiv 0$ and satisfies the following hypotheses

$$
\lim _{|s| \rightarrow 0} \frac{g(t, s, \lambda)}{s}=0
$$

uniformly for $t \in(0,1)$ and $\lambda$ on bounded sets.

Let $Y=C[0,1]$ with the norm $\|x\|_{\infty}=\max _{t \in[0,1]}|x(t)|$ and $E=\left\{x(t) \in C^{3}[0,1] \mid x(0)=x(1)=x^{\prime}(0)=x^{\prime}(1)=\right.$ $0\}$ with the norm $\|x\|=\max \left\{\|x\|_{\infty},\left\|x^{\prime}\right\|_{\infty},\left\|x^{\prime \prime}\right\|_{\infty},\left\|x^{\prime \prime \prime}\right\|_{\infty}\right\}$. Let $S_{k}^{+}$denote the set of functions in $E$ which have exactly $k-1$ interior nodal (i.e., nondegenerate) zeros in $(0,1)$ and are positive near $t=0$, set $S_{k}^{-}=-S_{k}^{+}$, and $S_{k}=S_{k}^{+} \cup S_{k}^{-}$. They are disjoint and open in $E$. Let $\Phi_{k}^{v}=\mathbb{R} \times S_{k}^{v}, v \in\{+,-\}$, and $\Phi_{k}=\mathbb{R} \times S_{k}$ under the product topology. Let $\mathcal{S}$ denote the closure in $\mathbb{R} \times E$ of the set of nontrivial solutions of (1) and let $\mathcal{S}^{ \pm}$denote the subset of $\mathcal{S}$ with $x \in \Phi_{k}^{ \pm}$and $\mathcal{S}^{ \pm}=\mathcal{S}^{+} \cup \mathcal{S}^{-}$.

Under condition (9), we will show that $\left(\mu_{k}, 0\right)$ is a bifurcation point of (8) and there are two distinct unbounded continua, $C_{k}^{+}$and $C_{k}^{-}$, consisting of the bifurcation branch $C_{k}$ from $\left(\mu_{k}, 0\right)$, where $\mu_{k}$ is the $k$ th eigenvalue of problem (2). Based on the above result, we investigate the existence of nodal solutions for problem (1).

Remark 2. By applying disconjugate operator theory $[25,26]$, the authors $[13,14,16]$ also established the spectrum structure of the corresponding linear eigenvalue problems. On the basis of the above spectrum structure, the authors $[13,14,16]$ studied the existence of nodal solutions of the above problem by applying Rabinowitz's global bifurcation theorem [8].

The rest of this paper is arranged as follows. In Section 2, we will establish unilateral global bifurcation results. In Section 3, we will investigate the existence of nodal solutions for problem (1) under the linear growth condition on $f$.

\section{Unilateral Global Bifurcation Results}

We define the linear operator $L: D(L) \subset E \rightarrow Y$

$$
L x=x^{\prime \prime \prime \prime}+k x^{\prime \prime}+l x, \quad x(t) \in D(L)
$$


with $D(L)=\left\{x(t) \in C^{4}[0,1] \mid x(0)=x(1)=x^{\prime}(0)=x^{\prime}(1)=\right.$ $0\}$.

From [31, p. 93], we consider the following auxiliary problem:

$$
\begin{aligned}
x^{\prime \prime \prime \prime}+k x^{\prime \prime}+l x & =e(t), \quad 0<t<1, \\
x(0) & =x(1)=x^{\prime}(0)=x^{\prime}(1)=0,
\end{aligned}
$$

for a given $e(t) \in C[0,1]$. We can get that problem (11) can be equivalently written as

$$
x(t)=L^{-1}(e)(t)=\int_{0}^{1} G(t, s) e(s) d s,
$$

where $G(t, s)>0$ was given in (2.29) of [31, p. 93].

Then $L$ is a closed operator and $L^{-1}: Y \rightarrow E$ is completely continuous.

Define the operator $H: \mathbb{R} \times E \rightarrow E$ by

$$
H(\lambda, x)(t):=\lambda L^{-1}(h x)+L^{-1}(g(t, x, \lambda)) .
$$

Furthermore, it is clear that problem (8) can be equivalently written as

$$
x=H(\lambda, x)(t) .
$$

Clearly, $H$ is completely continuous from $\mathbb{R} \times E \rightarrow E$ and $H(\lambda, 0)=0, \forall \lambda \in \mathbb{R}$.

$$
\text { Let }
$$

$$
\bar{g}(t, x, \lambda)=\max _{0 \leq|s| \leq x}|g(t, s, \lambda)|
$$

for $t \in(0,1), \lambda$ on bounded sets,

and then $\bar{g}$ is nondecreasing and

$$
\lim _{x \rightarrow 0^{+}} \frac{\bar{g}(t, x, \lambda)}{x}=0
$$

uniformly for $t \in(0,1)$ and $\lambda$ on bounded sets. Further it follows from (16) that

$$
\begin{aligned}
\frac{|g(t, x, \lambda)|}{\|x\|} & \leq \frac{\bar{g}(t, x, \lambda)}{\|x\|} \leq \frac{\bar{g}\left(t,\|x\|_{\infty}, \lambda\right)}{\|x\|} \\
& \leq \frac{\bar{g}(t,\|x\|, \lambda)}{\|x\|} \longrightarrow 0 \text { as }\|x\| \longrightarrow 0
\end{aligned}
$$

uniformly for $t \in(0,1)$ and $\lambda$ on bounded sets.

By (17), we have that $\left\|L^{-1}(g(t, x, \lambda))\right\| /\|x\| \rightarrow 0$ as $\|x\| \rightarrow 0$ uniformly for $t \in(0,1)$ and $\lambda$ on bounded sets. Furthermore, Applying Theorem 2 of [33], we may obtain the following result.

Theorem 3. Assume that (A1), (A2), and (9) hold. Then $\left(\lambda_{k}, 0\right)$ is a bifurcation point of problem (8) and there exist two distinct unbounded continua $C_{k}^{+}$and $C_{k}^{-}$of problem (8) emanating from $\left(\lambda_{k}, 0\right)$ such that either they are both unbounded or $C_{k}^{+} \cap C_{k}^{-} \neq\left\{\left(\lambda_{k}, 0\right)\right\}$.
Next, we prove that the first choice of the alternative of Theorem 3 is the only possibility. To do it, we give the following lemma.

Lemma 4. Let $C_{k}:=C_{k}^{+} \cup C_{k}^{-}$. If $C_{k} \subset \Phi_{k} \cup\left(\lambda_{k}, 0\right)$, then $C_{k}$ cannot contain a pair $(\bar{\lambda}, 0)$ and $\bar{\lambda} \neq \lambda_{k}$.

Proof. Suppose on the contrary that there exists $\left(\lambda_{m}, x_{m}\right) \rightarrow$ $\left(\lambda_{j}, 0\right)$ when $m \rightarrow+\infty$ with $\left(\lambda_{m}, x_{m}\right) \in C_{k}, x_{m} \neq 0$ and $j \neq k$. Let $y_{m}=x_{m} /\left\|x_{m}\right\|$; then $y_{m}$ should be a solution of problem

$$
y_{m}=L^{-1}\left(\lambda_{m} h y_{m}+\frac{g\left(t, x_{m}, \lambda\right)}{\left\|x_{m}\right\|}\right) .
$$

By (17), (18), and the compactness of $L^{-1}$ we obtain that for some convenient subsequence $y_{m} \rightarrow y_{0}$ as $m \rightarrow+\infty$. Now $y_{0}$ verifies the equation

$$
L y_{0}=\lambda_{j} h y_{0}
$$

and $\left\|y_{0}\right\|=1$. Hence $y_{0} \in S_{j}$ which is an open set in $E$, and as a consequence for some $m$ large enough, $y_{m} \in S_{j}$, and this is a contradiction.

Lemma 5. If $(\lambda, x)$ is a solution of (9) and $x \in \partial S_{k}$, then $x \equiv 0$.

Proof. By the proof of Theorem 3.1 in [16, p. 467] (see also Corollary 1.12 and the proof of Theorem 2.3, together with the remark following that proof, in [16]), we easily obtain the result.

Connecting Theorem 3 with Lemma 4, we can easily deduce the following Dancer-type unilateral global bifurcation result.

Theorem 6. Assume that (A1), (A2), and (9) hold; then $C_{k}^{+}$ and $C_{k}^{-}$are unbounded continua. Moreover, we have

$$
\begin{aligned}
& C_{k}^{+} \subset\left(\left\{\left(\lambda_{k}, 0\right)\right\} \cup\left(\mathbb{R} \times S_{k}^{+}\right)\right), \\
& C_{k}^{-} \subset\left(\left\{\left(\lambda_{k}, 0\right)\right\} \cup\left(\mathbb{R} \times S_{k}^{-}\right)\right) .
\end{aligned}
$$

Proof. By Theorem 3 with Lemma 4, we only prove $C_{k}^{v} \subset \Phi_{k}^{v} \cup$ $\left\{\left(\lambda_{k}, \theta\right)\right\}$ for $\nu \in\{+,-\}$. In the following, we only prove the case of $C_{k}^{+}$since the proof of $C_{k}^{-}$is similar.

We claim that there exists a neighborhood $B_{\delta}\left(\lambda_{k}, 0\right)$ of $\left(\lambda_{k}, 0\right)$ such that $\left(B_{\delta}\left(\lambda_{k}, 0\right) \cap C_{k}^{+}\right) \subset\left(\Phi_{k}^{+} \cup\left\{\left(\lambda_{k}, \theta\right)\right\}\right)$. Suppose on the contrary that there exists $\left(\lambda_{m}, x_{m}\right) \rightarrow\left(\lambda_{k}, 0\right)$ when $m \rightarrow+\infty$ with $\left(\lambda_{m}, x_{m}\right) \in C_{k}^{+} \backslash\left(\mathbb{R} \times S_{k}^{+}\right)$and $x_{m} \neq 0$. Let $z_{m}=x_{m} /\left\|x_{m}\right\|$; then $z_{m}$ should be a solution of problem

$$
z_{m}=L^{-1}\left(\lambda_{m} h z_{m}+\frac{g\left(t, x_{m}, \lambda_{m}\right)}{\left\|x_{m}\right\|}\right) .
$$

By (17), (21), and the compactness of $L^{-1}$, we obtain that for some convenient subsequence $z_{m} \rightarrow z_{0}$ as $m \rightarrow+\infty$. Now $z_{0}$ verifies the equation

$$
L z_{0}=\lambda_{k} h z_{0}
$$


and $\left\|z_{0}\right\|=1$. Hence $z_{0} \in S_{k}$ which is an open set in $E$, and as a consequence for some $m$ large enough, $x_{m} \in S_{k}^{+}$, and this is a contradiction.

Suppose that $C_{k}^{+} \nsubseteq \Phi_{k}^{+} \cup\left\{\left(\lambda_{k}, \theta\right)\right\}$. Then there exists $\left(\lambda^{*}, x\right) \in C_{k}^{+} \cap\left(\mathbb{R} \times \partial S_{k}^{+}\right)$such that $\left(\lambda^{*}, x\right) \neq\left(\lambda_{k}, \theta\right)$ and $\left(\lambda_{n}, x_{n}\right) \rightarrow\left(\lambda^{*}, x\right)$ with $\left(\lambda_{n}, x_{n}\right) \in C_{k}^{+} \cap\left(\mathbb{R} \times S_{k}^{+}\right)$. Since $x \in \partial S_{k}^{+}$, by Lemma $8, x \equiv 0$. Let $y_{n}:=x_{n} /\left\|x_{n}\right\| ;$; then $y_{n}$ should be a solution of problem

$$
y_{n}=\lambda_{n} L^{-1}\left[\lambda h y_{n}+\frac{g\left(t, x_{n}, \lambda_{n}\right)}{\left\|x_{n}\right\|}\right] .
$$

By (17), (23), and the compactness of $L^{-1}$, we obtain that for some convenient subsequence $y_{n} \rightarrow y_{0} \neq 0$ as $n \rightarrow+\infty$. Now $y_{0}$ verifies the equation

$$
L y_{0}=\lambda^{*} h(t) y_{0}(t), \quad t \in(0,1)
$$

and $\left\|y_{0}\right\|=1$. Hence $\lambda^{*}=\lambda_{i}$, for some $i \neq k, i \in \mathbb{N}$. Therefore, $\left(\lambda_{n}, x_{n}\right) \rightarrow\left(\lambda_{i}, \theta\right)$ with $\left(\lambda_{n}, x_{n}\right) \in C_{k}^{+} \cap\left(\mathbb{R} \times S_{k}^{+}\right)$. This contradicts Lemma 4.

In order to treat the case $f_{0} \notin(0,+\infty)$ or $f_{\infty} \notin(0,+\infty)$, we will need the following results.

Definition 7 (see [38]). Let $X$ be a Banach space and let $\left\{C_{n} \mid\right.$ $n=1,2, \ldots\}$ be a family of subsets of $X$. Then the superior limit $\mathbb{D}$ of $\left\{C_{n}\right\}$ is defined by

$$
\begin{aligned}
\mathbb{D} & :=\limsup _{n \rightarrow \infty} C_{n}=\left\{x \in X \mid \exists\left\{n_{i}\right\} \subset \mathbb{N}, x_{n_{i}}\right. \\
& \left.\in C_{n_{i}} \text {, such that } x_{n_{i}} \longrightarrow x\right\} .
\end{aligned}
$$

Lemma 8 (see [38]). Each connected subset of metric space $X$ is contained in a component, and each connected component of $X$ is closed.

Lemma 9 (see [39]). Let $X$ be a Banach space and let $\left\{C_{n} \mid n=\right.$ $1,2, \ldots\}$ be a family of closed connected subsets of $X$. Assume that

(i) there exist $z_{n} \in C_{n}, n=1,2, \ldots$, and $z^{*} \in X$, such that $z_{n} \rightarrow z^{*}$

(ii) $r_{n}=\sup \left\{\|x\| \mid x \in C_{n}\right\}=\infty$;

(iii) for all $R>0,\left(\bigcup_{n=1}^{\infty} C_{n}\right) \cap B_{R}$ is a relative compact set of $X$, where

$$
B_{R}=\{x \in X \mid\|x\| \leq R\} .
$$

Then there exists an unbounded component $C$ in $\mathbb{D}$ and $z^{*} \in C$.

Lemma 10. Assume (A1) and (A2). Let $g_{n} \in C([0,1],(0$, $+\infty)$ ). Assume that $I$ is a subset of $[0,1]$ with meas $(I)>0$, and let

$$
\lim _{n \rightarrow+\infty} g_{n}(t)=+\infty
$$

uniformly on I. Let $y_{n}$ be a solution of the equation

$$
\begin{aligned}
y_{n}^{\prime \prime \prime \prime}+k y_{n}^{\prime \prime}+l y_{n} & =\lambda h(t) g_{n}(t) y_{n}, \quad 0<t<1, \\
y_{n}(0) & =y_{n}(1)=y_{n}^{\prime}(0)=y_{n}^{\prime}(1)=0,
\end{aligned}
$$

and then $y_{n}$ must change sign on $I$ as $n$ is large enough.
Proof. After taking a subsequence if necessary, we may assume that

$$
h(t) g_{n_{j}}(t) \geq j, \quad t \in I,
$$

for $j$ large enough. By [32, Lemma 2.4], $L(x)=0$ is disconjugate on $[0,1]$, which is a key condition in Elias [25]. Obviously, $y_{n}$ have the property $P$. (For the definition of property $P$, see $[25$, p. 36].) Now, from the proof of $[25$, Lemma 4] (see also the remarks in the final paragraph in [25, p. 43]; or see the proof of [16, Lemma 3.7]), it follows that, for all $n$ sufficiently large, $y_{n}$ must change sign on $I$.

\section{Main Results}

In this section, we first study the following eigenvalue problem:

$$
\begin{array}{r}
x^{\prime \prime \prime \prime}+k x^{\prime \prime}+l x=\lambda r h(t) f(x), \quad 0<t<1, \\
x(0)=x(1)=x^{\prime}(0)=x^{\prime}(1)=0,
\end{array}
$$

where $\lambda>0$ is a parameter.

In the section, $f \in C(\mathbb{R}, \mathbb{R})$ satisfy the following conditions:

(H1) $s f(s)>0$ for $s \neq 0$.

(H2) $f_{0}, f_{\infty} \in(0,+\infty)$.

(H3) $f_{0} \in(0, \infty)$ and $f_{\infty}=\infty$.

(H4) $f_{0}=\infty$ and $f_{\infty} \in(0, \infty)$.

(H5) $f_{0} \in(0, \infty)$ and $f_{\infty}=0$.

(H6) $f_{0}=0$ and $f_{\infty} \in(0, \infty)$.

(H7) $f_{0}=0$ and $f_{\infty}=\infty$.

(H8) $f_{0}=\infty$ and $f_{\infty}=0$.

(H9) $f_{0}=\infty$ and $f_{\infty}=\infty$.

(H10) $f_{0}=0$ and $f_{\infty}=0$,

where

$$
\begin{aligned}
f_{0} & =\lim _{|x| \rightarrow 0} \frac{f(x)}{x}, \\
f_{\infty} & =\lim _{|x| \rightarrow \infty} \frac{f(x)}{x} .
\end{aligned}
$$

Let $\zeta(x), \xi(x) \in C(\mathbb{R}, \mathbb{R})$ be such that

$$
\begin{aligned}
& f(x)=f_{0} x+\zeta(x), \\
& f(x)=f_{\infty} x+\xi(x)
\end{aligned}
$$

with

$$
\begin{aligned}
& \lim _{|x| \rightarrow 0} \frac{\zeta(x)}{x}=0, \\
& \lim _{|x| \rightarrow \infty} \frac{\xi(x)}{x}=0 .
\end{aligned}
$$


Let us consider

$$
\begin{gathered}
x^{\prime \prime \prime \prime}+k x^{\prime \prime}+l x=\lambda r h(t) f_{0} x+\lambda r h(t) \zeta(x), \\
\quad 0<t<1, \\
x(0)=x(1)=x^{\prime}(0)=x^{\prime}(1)=0
\end{gathered}
$$

as a bifurcation problem from the trivial solution $x \equiv 0$ and

$$
x^{\prime \prime \prime \prime}+k x^{\prime \prime}+l x=\lambda r h(t) f_{\infty} x+\lambda r h(t) \xi(x),
$$$$
0<t<1
$$

$$
x(0)=x(1)=x^{\prime}(0)=x^{\prime}(1)=0
$$

as a bifurcation problem from infinity.

We add the points $\{(\lambda, \infty) \mid \lambda \in \mathbb{R}\}$ to space $\mathbb{R} \times E$. By [40], we note that problem (34) and problem (35) are the same, and each of them is equivalent to problem (30). By Theorems 3 and 6 and the results of Rabinowitz [41], we have the following Lemma.

Lemma 11. Let (A1), (A2), (H1), and (H2) hold. $\left(\lambda_{k} / r f_{0}, 0\right)$ and $\left(\lambda_{k} / r f_{\infty}, \infty\right)$ are bifurcation points for problem (30). Moreover, there are two distinct unbounded subcontinua of solutions to problem (30), $\mathscr{D}_{k}^{+}$and $\mathscr{D}_{k}^{-}$, consisting of the bifurcation branch $\mathscr{D}_{k}^{v}$ emanating from $\left(\lambda_{k} / r f_{0}, 0\right)$ or $\left(\lambda_{k} / r f_{\infty}, \infty\right)$. For $v=+,-, \mathscr{D}_{k}^{v}$ joins $\left(\lambda_{k} / r f_{0}, 0\right)$ to $\left(\lambda_{k} / r f_{\infty}, \infty\right)$, such that $\mathscr{D}_{k}^{v} \subset\left(\Phi_{k}^{v} \cup\left\{\left(\lambda_{k} / r f_{0}, 0\right)\right\}\right)$ and $\mathscr{D}_{k}^{v} \subset\left(\Phi_{k}^{v} \cup\left\{\left(\lambda_{k} / r f_{\infty}, \infty\right)\right\}\right)$.

Remark 12. Any solution of the problem (30) of the form $(1, x)$ yields a solution $x$ of the problem (1). In order to prove our main results, one will only show that $\mathscr{D}_{k}^{v}$ crosses the hyperplane $\{1\} \times E$ in $\mathbb{R} \times E$.

Theorem 13. Let (A1), (A2), (H1), and (H2) hold, and either $\lambda_{k} / f_{\infty}<r<\lambda_{k} / f_{0}$ or $\lambda_{k} / f_{0}<r<\lambda_{k} / f_{\infty}$. Then problem (1) has two solutions $x_{k}^{+}$and $x_{k}^{-}, x_{k}^{+}$has exactly $k-1$ simple zeros in $(0,1)$ and is positive near $t=0$, and $x_{k}^{-}$has exactly $k-1$ simple zeros in $(0,1)$ and is negative near $t=0$.

Proof of Theorem 13. By Lemma 11 and Remark 12, we only prove $\mathscr{D}_{k}^{+}$and $\mathscr{D}_{k}^{-}$crosses the hyperplane $\{1\} \times E$ in $\mathbb{R} \times E$. We only prove the case of $\mathscr{D}_{k}^{+}$since the case of $\mathscr{D}_{k}^{-}$is similar.

Case 1. (i) Consider $\lambda_{k} / f_{\infty}<r<\lambda_{k} / f_{0}$.

In this case, we only need to show that

$$
\left(\frac{\lambda_{k}}{r f_{\infty}}, \frac{\lambda_{k}}{r f_{0}}\right) \subseteq\left\{\mu \in \mathbb{R}:(\mu, x) \in \mathscr{D}_{k}^{+}\right\} .
$$

We divide the proof into two steps.

Let $\left(\lambda_{n}, x_{n}\right) \in \mathscr{D}_{k}^{+}$satisfy

$$
\lambda_{n}+\left\|x_{n}\right\| \longrightarrow \infty
$$

We note that $\lambda_{n}>0$ for all $n \in \mathbb{N}$, since $(0,0)$ is the only solution of the problem (30) for $\lambda=0$ and $\mathscr{D}_{k}^{+} \cap(\{0\} \times E)=\emptyset$.

Step 1. We show that if there exists a constant number $M>0$ such that

$$
\lambda_{n} \in(0, M]
$$

for $n \in \mathbb{N}$ large enough, then $\mathscr{D}_{k}^{+}$joins $\left(\lambda_{k} / r f_{0}, 0\right)$ to $\left(\lambda_{k} /\right.$ $\left.r f_{\infty}, \infty\right)$.

In this case, it follows that

$$
\left\|x_{n}\right\| \longrightarrow \infty
$$

By (32) and (33), let

$$
\bar{\xi}(x)=\max \{|\xi(s)|: 0 \leq|s| \leq x\},
$$

and then $\bar{\xi}$ is nondecreasing and

$$
\lim _{x \rightarrow \infty} \frac{\bar{\xi}(x)}{x}=0 .
$$

We divide the equation

$$
L x_{n}=\lambda_{n} r a(t) x_{n}+\lambda_{n} r \xi\left(x_{n}\right)
$$

by $\left\|x_{n}\right\|$ and set $y_{n}=x_{n} /\left\|x_{n}\right\|$. Since $y_{n}$ is bounded in $E$, after taking a subsequence if necessary, we have that $y_{n} \rightarrow y$ for some $y \in E$ with $\|y\|=1$. Moreover, from (41) and the fact that $\bar{\xi}$ is nondecreasing, we have that

$$
\lim _{n \rightarrow \infty} \frac{\left|\xi\left(x_{n}(t)\right)\right|}{\left\|x_{n}\right\|}=0,
$$

since

$$
\begin{aligned}
\frac{\left|\xi\left(x_{n}(t)\right)\right|}{\left\|x_{n}\right\|} & \leq \frac{\bar{\xi}\left(\left|x_{n}(t)\right|\right)}{\left\|x_{n}\right\|} \leq \frac{\bar{\xi}\left(\left\|x_{n}(t)\right\|_{\infty}\right)}{\left\|x_{n}\right\|} \\
& \leq \frac{\bar{\xi}\left(\left\|x_{n}(t)\right\|\right)}{\left\|x_{n}\right\|} .
\end{aligned}
$$

By the continuity and compactness of $L^{-1}$, it follows that

$$
y^{\prime \prime \prime \prime}+k y^{\prime \prime}+l y=\bar{\lambda} r a(t) f_{\infty} y,
$$

where $\bar{\lambda}:=\lim _{n \rightarrow \infty} \lambda_{n}$, again choosing a subsequence and relabeling if necessary.

We claim that $y \in \mathscr{D}_{k}^{+}$.

It is clear that $y \in \overline{C_{k}^{+}} \subset C_{k}^{+}$since $\mathscr{D}_{k}^{+}$is closed in $\mathbb{R} \times E$. Thus, $\bar{\lambda} r a(t) f_{\infty}=\lambda_{k}$, so that

$$
\bar{\lambda}=\frac{\lambda_{k}}{r f_{\infty}}
$$

Therefore, $\mathscr{D}_{k}^{+}$joins $\left(\lambda_{k} / r f_{0}, 0\right)$ to $\left(\lambda_{k} / r f_{\infty}, \infty\right)$.

Step 2. We show that there exists a constant $M$ such that $\lambda_{n} \epsilon$ $(0, M]$ for all $n$. On the contrary, choosing a subsequence and relabeling if necessary, it follows that

$$
\lim _{n \rightarrow \infty} \lambda_{n}=\infty
$$

Since $\left(\lambda_{n}, x_{n}\right) \in \mathscr{D}_{k}^{+}$, it follows that

$$
x_{n}^{\prime \prime \prime \prime}+k x_{n}^{\prime \prime}+l x_{n}=\lambda_{n} r h(t) f\left(x_{n}\right) .
$$


Let

$$
0<\tau(1, n)<\tau(2, n)<\cdots<\tau(k-1, n)<1
$$

denote the simple zeros of $x_{n}(t)$ in $(0,1)$. Let $\tau(0, n)=0$ and $\tau(k, n)=1$. Then, after taking a subsequence if necessary,

$$
\lim _{n \rightarrow+\infty} \tau(j, n)=\tau(j, \infty), \quad j=0,1, \ldots, k
$$

We claim that there exists $j_{0} \in\{0,1, \ldots, k\}$ such that

$$
\tau\left(j_{0}, \infty\right)<\tau\left(j_{0}+1, \infty\right)
$$

Otherwise, we have

$$
\begin{gathered}
1=\sum_{j=0}^{k-1}(\tau(j+1, n)-\tau(j, n)) \longrightarrow \\
\sum_{j=0}^{k-1}(\tau(j+1, \infty)-\tau(j, \infty))=0 .
\end{gathered}
$$

This is a contradiction. Let $(a, b) \subset\left(\tau\left(j_{0}, \infty\right), \tau\left(j_{0}+1, \infty\right)\right)$ with $a<b$. For all $n$ sufficiently large, we have $(a, b) \subset$ $\left(\tau\left(j_{0}, n\right), \tau\left(j_{0}+1, n\right)\right)$. So $x_{n}(t)$ does not change its sign in $(a, b)$.
On the other hand, let

$$
x_{n}^{\prime \prime \prime \prime}+k x_{n}^{\prime \prime}+l x_{n}=\lambda_{n} r h(t) \tilde{f}_{n}(t)
$$

where

$$
\tilde{f}_{n}(t)= \begin{cases}\frac{f\left(x_{n}\right)}{x_{n}}, & x_{n}(t) \neq 0, \\ f_{0}, & x_{n}(t)=0 .\end{cases}
$$

Conditions $(H 1)$ and $(H 2)$ imply that there exists a positive constant $Q>0$ such that $h(t) \tilde{f}_{n}(t)>Q$ for any $t \in(a, b)$ and all $n \in \mathbb{N}$. By Lemma 10 , we get that $x_{n}$ must change its sign in $(a, b)$ for $n$ large enough, which is the contradicts. Therefore,

$$
\lambda_{n} \leq M
$$

for some constant number $M>0$ and $n \in \mathbb{N}$ sufficiently large.

Case 2. (ii) Consider $\lambda_{k} / f_{0}<r<\lambda_{k} / f_{\infty}$.

The proof is similar to that for Case 1 , so we omit it.

Theorem 14. Let (A1), (A2), (H1), and (H3) hold. Assume condition $r \in\left(0, \lambda_{k} / f_{\infty}\right)$ holds for some $k \in \mathbb{N}$. Then problem (1) has two solutions $x_{k}^{+}$and $x_{k}^{-}, x_{k}^{+}$has exactly $k-1$ simple zeros in $(0,1)$ and is positive near $t=0$, and $x_{k}^{-}$has exactly $k-1$ simple zeros in $(0,1)$ and is negative near $t=0$.

Proof. Inspired by the idea of [42], we define the cut-off function of $f$ as the following:

$$
f^{[n]}(s):= \begin{cases}n s, & s \in(-\infty,-2 n] \cup[2 n,+\infty), \\ \frac{2 n^{2}+f(-n)}{n}(s+n)+f(-n), & s \in(-2 n,-n), \\ \frac{2 n^{2}-f(n)}{n}(s-n)+f(n), & s \in(n, 2 n), \\ f(s), & s \in[-n, n] .\end{cases}
$$

We consider the following problem:

$$
\begin{aligned}
x^{\prime \prime \prime \prime}+k x^{\prime \prime}+l x & =\lambda r h(t) f^{[n]}(x), \quad 0<t<1, \\
x(0) & =x(1)=x^{\prime}(0)=x^{\prime}(1)=0 .
\end{aligned}
$$

Clearly, we can see that $\lim _{n \rightarrow+\infty} f^{[n]}(s)=f(s),\left(f^{[n]}\right)_{0}=$ $f_{0}$, and $\left(f^{[n]}\right)_{\infty}=n$.

Similar to the proof of Theorem 13, by Lemma 11 and Remark 12, there are two distinct unbounded subcontinua of solutions to problem (57), $\mathscr{D}_{k}^{+[n]}$ and $\mathscr{D}_{k}^{-[n]}$ emanating from $\left(\lambda_{k} / r f_{0}, 0\right)$, and joins to $\left(\lambda_{k} / r n, \infty\right)$.

Taking $z_{n}=\left(\lambda_{k} / r n, \infty\right)$ and $z^{*}=(0, \infty)$, we have that $z_{n} \rightarrow z^{*}$.

So condition (i) in Lemma 9 is satisfied with $z^{*}=(0, \infty)$. Obviously

$$
r_{n}=\sup \left\{\lambda+\|u\| \mid(\lambda, u) \in \mathscr{D}_{k}^{v[n]}\right\}=\infty,
$$

and accordingly, (ii) in Lemma 9 holds. (iii) in Lemma 9 can be deduced directly from the Arzela-Ascoli Theorem and the definition of $f^{[n]}$.

Therefore, by Lemma 9, lim $\sup _{n \rightarrow \infty} \mathscr{D}_{k}^{\gamma[n]}$ contains an unbounded component $\mathscr{D}_{k}^{v}$ emanating from $\left(\lambda_{k} / r f_{0}, 0\right)$, and $\mathscr{D}_{k}^{v}$ joins $\left(\lambda_{k} / r f_{0}, 0\right)$ to $(0, \infty)$.

From $\lim _{n \rightarrow+\infty} f^{[n]}(s)=f(s)$, (57) can be converted to the equivalent equation (30). Thus, $\mathscr{D}_{k}^{v}$ is an unbounded component of solutions of problem (30) emanating from $\left(\lambda_{k} / r f_{0}, 0\right)$, and $\mathscr{D}_{k}^{v}$ joins $\left(\lambda_{k} / r f_{0}, 0\right)$ to $(0, \infty)$. We can prove the result.

Theorem 15. Let (A1), (A2), (H1), and (H4) hold. Assume that condition $r \in\left(0, \lambda_{k} / f_{0}\right)$ holds for some $k \in \mathbb{N}$. Then problem (1) has two solutions $x_{k}^{+}$and $x_{k}^{-}, x_{k}^{+}$has exactly $k-1$ simple zeros in $(0,1)$ and is positive near $t=0$, and $x_{k}^{-}$ has exactly $k-1$ simple zeros in $(0,1)$ and is negative near $t=0$. 
Proof. If $(\lambda, x)$ is any nontrivial solution of problem (30), dividing problem (30) by $\|x\|^{2}$ and setting $y=x /\|x\|^{2}$ yield

$$
\begin{array}{r}
y^{\prime \prime \prime \prime}+k y^{\prime \prime}+l y=\lambda r h(t) \frac{f(x)}{\|x\|^{2}}, \quad 0<t<1, \\
y(0)=y(1)=y^{\prime}(0)=y^{\prime}(1)=0 .
\end{array}
$$

Define

$$
\tilde{f}(y):= \begin{cases}\|y\|^{2} f\left(\frac{y}{\|y\|^{2}}\right), & \text { if } y \neq 0, \\ 0, & \text { if } y=0 .\end{cases}
$$

Evidently, problem (59) is equivalent to

$$
\begin{array}{r}
y^{\prime \prime \prime \prime}+k y^{\prime \prime}+l y=\lambda r h(t) \tilde{f}(y), \quad 0<t<1, \\
y(0)=y(1)=y^{\prime}(0)=y^{\prime}(1)=0 .
\end{array}
$$

It is obvious that $(\lambda, 0)$ is always the solution of problem (59). By simple computation, we can show that $\widetilde{f}_{0}=f_{\infty} \in$ $(0, \infty)$ and $\widetilde{f}_{\infty}=f_{0}=\infty$. Now, applying Theorem 13, there are two distinct unbounded subcontinua of solutions to problem (61), $\mathscr{C}_{k}^{+}$and $\mathscr{C}_{k}^{-}$emanating from $\left(\lambda^{\nu} / r \widetilde{f}_{0}, 0\right)$, and joins to $(0, \infty)$.

Under the inversion $y \rightarrow y /\|y\|^{2}=x$, we obtain $\mathscr{C}_{k}^{v} \rightarrow$ $\mathscr{D}_{k}^{v}$ being an unbounded component of solutions of problem (30) emanating from $(0,0)$, and joins to $\left(\lambda^{\nu} / r f_{\infty}, \infty\right)$.

Moreover, by Remark 12 and the problem (1), we can obtain that $\mathscr{D}_{k}^{\nu} \subset \mathcal{S}_{k}^{\nu}$.

Thus, $\mathscr{D}_{k}^{v}$ is an unbounded component of solutions of problem (1) such that $\mathscr{D}_{k}^{v}$ joins $(0,0)$ to $\left(\lambda^{\nu} / r f_{\infty}, \infty\right)$.

Theorem 16. Let (A1), (A2), (H1), and (H5) hold. Assume that condition $r \in\left(\lambda_{k} / f_{0},+\infty\right)$ holds. Then problem (1) has two solutions $x_{k}^{+}$and $x_{k}^{-}, x_{k}^{+}$has exactly $k-1$ simple zeros in $(0,1)$ and is positive near $t=0$, and $x_{k}^{-}$has exactly $k-1$ simple zeros in $(0,1)$ and is negative near $t=0$.

Proof. In view of the proof to prove Theorem 13, we only need to show that $\mathscr{D}_{k}^{v}$ joins $\left(\lambda_{k} / r f_{0}, 0\right)$ to $(\infty, \infty)$. To do this, it is enough to prove that $\left[\lambda_{k} / r f_{0},+\infty\right) \subset \operatorname{Proj}_{R} \mathscr{D}_{k}^{\nu}$.

Assume on the contrary that $\sup \left\{\lambda \mid(\lambda, u) \in \mathscr{D}_{k}^{\nu}\right\}<+\infty$, and then there exists a sequence $\left(\mu_{n}, x_{n}\right) \in \mathscr{D}_{k}^{v}$ such that

$$
\begin{aligned}
\lim _{n \rightarrow \infty}\left\|x_{n}\right\| & =+\infty, \\
\mu_{n} & \leq c_{0}
\end{aligned}
$$

for some positive constant $c_{0}$ depending not on $n$.

By $(H 3)$, let $\bar{f}(x)=\max _{0 \leq|s| \leq x}|f(s)|$, and then $\bar{f}$ is nondecreasing and

$$
\lim _{x \rightarrow+\infty} \frac{\bar{f}(x)}{x}=0
$$

We consider the equation

$$
\begin{array}{r}
x_{n}^{\prime \prime \prime \prime}+k x_{n}^{\prime \prime}+l x_{n}=\mu_{n} r h(t) f\left(x_{n}\right), \quad 0<t<1, \\
x_{n}(0)=x_{n}(1)=x_{n}^{\prime}(0)=x_{n}^{\prime}(1)=0 .
\end{array}
$$

Let $y_{n}=x_{n} /\left\|x_{n}\right\|$, and $y_{n}$ should be the solutions of problem

$$
\begin{array}{r}
y_{n}^{\prime \prime \prime \prime}+k y_{n}^{\prime \prime}+l y_{n}=\mu_{n} r h(t) \frac{f\left(x_{n}\right)}{\left\|x_{n}\right\|}, \quad 0<t<1, \\
y_{n}(0)=y_{n}(1)=y_{n}^{\prime}(0)=y_{n}^{\prime}(1)=0 .
\end{array}
$$

Since $y_{n}$ is bounded in $E$, choosing a subsequence and relabeling if necessary, we have that $y_{n} \rightarrow y$ for some $y \in E$ and $\|y\|=1$.

Furthermore, from (63) and the fact that $\bar{f}$ is nondecreasing, we have that

$$
\lim _{n \rightarrow \infty} \frac{f\left(x_{n}\right)}{\left\|x_{n}\right\|}=0
$$

since

$$
\begin{aligned}
\frac{f\left(x_{n}\right)}{\left\|x_{n}\right\|} \leq \frac{\bar{f}\left(\left|x_{n}\right|\right)}{\left\|x_{n}\right\|} \leq \frac{\bar{f}\left(\left\|x_{n}\right\|_{\infty}\right)}{\left\|x_{n}\right\|} \leq \frac{\bar{f}\left(\left\|x_{n}\right\|\right)}{\left\|x_{n}\right\|} & \longrightarrow 0, \\
n & \longrightarrow+\infty .
\end{aligned}
$$

By (12), (65), (66), and the compactness of $L^{-1}$, we obtain that $y(t) \equiv 0, \forall t \in[0,1]$.

This contradicts $\|y(t)\|=1$.

Theorem 17. Let (A1), (A2), (H1), and (H6) hold. Assume that condition $r \in\left(\lambda_{k} / f_{\infty},+\infty\right)$ holds. Then problem (1) has two solutions $x_{k}^{+}$and $x_{k}^{-}, x_{k}^{+}$has exactly $k-1$ simple zeros in $(0,1)$ and is positive near $t=0$, and $x_{k}^{-}$has exactly $k-1$ simple zeros in $(0,1)$ and is negative near $t=0$.

Proof. Similar to the method of the proof of Theorem 15 and the conclusions of Theorem 16, we can prove the conclusion.

Theorem 18. Let (A1), (A2), (H1), and (H7) hold. Assume that condition $r \in(0,+\infty)$ holds. Then problem (1) has two solutions $x_{k}^{+}$and $x_{k}^{-}, x_{k}^{+}$has exactly $k-1$ simple zeros in $(0,1)$ and is positive neart $=0$, and $x_{k}^{-}$has exactly $k-1$ simple zeros in $(0,1)$ and is negative near $t=0$. 
Proof. Define

$$
f^{[n]}(s):= \begin{cases}n s, & s \in(-\infty,-2 n] \cup[2 n,+\infty), \\ \frac{2 n^{2}+f(-n)}{n}(s+n)+f(-n), & s \in(-2 n,-n), \\ f(s), & s \in(n, 2 n), \\ -\left[f\left(-\frac{2}{n}\right)+\frac{1}{n^{2}}\right](n s+2)+f\left(-\frac{2}{n}\right), & s \in\left(-\frac{2}{n},-\frac{1}{n}\right), \\ {\left[f\left(\frac{2}{n}\right)-\frac{1}{n^{2}}\right](n s-2)+f\left(\frac{2}{n}\right),} & s \in\left(\frac{1}{n}, \frac{2}{n}\right), \\ \frac{1}{n} s, & s \in\left[-\frac{1}{n}, \frac{1}{n}\right] .\end{cases}
$$

We consider the following problem:

$$
\begin{array}{r}
x^{\prime \prime \prime \prime}+k x^{\prime \prime}+l x=\lambda r h(t) f^{[n]}(x), \quad 0<t<1, \\
x(0)=x(1)=x^{\prime}(0)=x^{\prime}(1)=0 .
\end{array}
$$

Clearly, we can see that $\lim _{n \rightarrow+\infty} f^{[n]}(s)=f(s),\left(f^{[n]}\right)_{0}=$ $1 / n$, and $\left(f^{[n]}\right)_{\infty}=n$.

Applying the similar method used in the proof of Theorem 13, by Lemma 11 and Remark 12, there are two distinct unbounded subcontinua of solutions to problem (69), $\mathscr{D}_{k}^{+[n]}$ and $\mathscr{D}_{k}^{-[n]}$ emanating from $\left(n \lambda_{k} / r, 0\right)$ or $\left(\lambda_{k} / r n, \infty\right)$, and joins $\left(n \lambda_{k} / r, 0\right)$ to $\left(\lambda_{k} / r n, \infty\right)$.

Taking $z_{n}=\left(n \lambda_{k} / r, 0\right)$ and $z^{*}=(\infty, 0)$ or $z_{n}=$ $\left(\lambda_{k} / r n, \infty\right)$ and $z^{*}=(0, \infty)$, we have that $z_{n} \rightarrow z^{*}$. By Lemma 9, we obtain that $\lim \sup _{n \rightarrow \infty} \mathscr{D}_{k}^{v[n]}$ contains an unbounded component $\mathscr{D}_{k}^{v}$ with $(\infty, 0),(0, \infty) \in \mathscr{D}_{k}^{v}$.

From $\lim _{n \rightarrow+\infty} f^{[n]}(s)=f(s)$, (69) can be converted to the equivalent equation (30). Thus, $\mathscr{D}_{k}^{v}$ is an unbounded component of solutions of problem (30) emanating from $(\infty, 0)$ or $(0, \infty)$ and joins $(\infty, 0)$ to $(0, \infty)$. $\delta_{k}^{v}$

Moreover, by Remark 12 and (1), we can obtain that $\mathscr{D}_{k}^{v} \subset$

Thus, $\mathscr{D}_{k}^{\nu}$ is an unbounded component of solutions of problem (1) emanating from $(\infty, 0)$ or $(0, \infty)$ and joins $(\infty, 0)$ to $(0, \infty)$.

Theorem 19. Let (A1), (A2), (H1), and (H8) hold. Assume that condition $r \in(0,+\infty)$ holds. Then problem (2) has two solutions $x_{k}^{+}$and $x_{k}^{-}, x_{k}^{+}$has exactly $k-1$ simple zeros in $(0,1)$ and is positive near $t=0$, and $x_{k}^{-}$has exactly $k-1$ simple zeros in $(0,1)$ and is negative near $t=0$.

Proof. Similar to the method of the proof of Theorem 14 and the conclusions of Theorem 18, we can obtain the desired results.

Theorem 20. Let (A1), (A2), (H1), and (H9) hold. There exists $\lambda_{k}^{+}>0$ such that $r \in\left(0, \lambda_{k}^{+}\right)$. Then problem (1) has two solutions $x_{k}^{+}$and $x_{k}^{-}, x_{k}^{+}$has exactly $k-1$ simple zeros in $(0,1)$ and is positive near $t=0$, and $x_{k}^{-}$has exactly $k-1$ simple zeros in $(0,1)$ and is negative near $t=0$.

Proof. Define

$$
f^{[n]}(s):= \begin{cases}\frac{n s,}{2 n^{2}+f(-n)} & s \in(-\infty,-2 n] \cup[2 n,+\infty), \\ \frac{2 n^{2}-f(n)}{n}(s-n)+f(n), & s \in(-2 n,-n), \\ f(s), & s \in\left[-n,-\frac{2}{n}\right] \cup\left[\frac{2}{n}, n\right], \\ -\left[f\left(-\frac{2}{n}\right)+1\right](n s+2)+f\left(-\frac{2}{n}\right), & s \in\left(-\frac{2}{n},-\frac{1}{n}\right), \\ {\left[f\left(\frac{2}{n}\right)-1\right](n s-2)+f\left(\frac{2}{n}\right),} & s \in\left(\frac{1}{n}, \frac{2}{n}\right), \\ n s, & s \in\left[-\frac{1}{n}, \frac{1}{n}\right] .\end{cases}
$$


We consider the following problem:

$$
\begin{array}{r}
x^{\prime \prime \prime \prime}+k x^{\prime \prime}+l x=\lambda r h(t) f^{[n]}(x), \quad 0<t<1, \\
x(0)=x(1)=x^{\prime}(0)=x^{\prime}(1)=0 .
\end{array}
$$

It is of no difficulty to verify that $\lim _{n \rightarrow+\infty} f^{[n]}(s)=f(s)$, $\left(f^{[n]}\right)_{0}=n$, and $\left(f^{[n]}\right)_{\infty}=n$.

Theorem 18 implies that there exist two sequences of unbounded continua of solutions to problem (71), $\mathscr{D}_{k}^{+[n]}$ and $\mathscr{D}_{k}^{-[n]}$ emanating from $\left(\lambda_{k} / r n, 0\right)$ or $\left(\lambda_{k} / r n, \infty\right)$.

By making use of Lemma 9 again, we obtain that there exist two unbounded components $\mathscr{D}_{k}^{+}$and $\mathscr{D}_{k}^{-}$of $\lim \sup _{n \rightarrow \infty} \mathscr{D}_{k}^{\nu[n]}$ such that $(0,0) \in \mathscr{D}_{k}^{\nu}$ and $(0, \infty) \in \mathscr{D}_{k}^{\nu}$.

Theorem 21. Let $(A 1),(A 2),(H 1)$, and (H10) hold. There exists $\lambda_{k}^{+}>0$, such that $r \in\left(\lambda_{k}^{+},+\infty\right)$. Then problem (1) has two solutions $x_{k}^{+}$and $x_{k}^{-}, x_{k}^{+}$has exactly $k-1$ simple zeros in $(0,1)$ and is positive near $t=0$, and $x_{k}^{-}$has exactly $k-1$ simple zeros in $(0,1)$ and is negative near $t=0$.

Proof. Similar to the method of the proof of Theorem 14 and the conclusions of Theorem 20, we can obtain the desired results.

Using the similar proof with that of Theorems 13-16, we can obtain the result.

Theorem 22. Let (A1), (A2), (H1), and (H3) hold. Assume that the following condition holds for some $k, n \in \mathbb{N}$ with $k \leq n$ :

$$
r<\frac{\lambda_{k}}{f_{\infty}}<\frac{\lambda_{n}}{f_{\infty}}
$$

Then problem (1) possesses $n-k+1$ pairs solutions $x_{i}^{+}$and $x_{i}^{-}$ for $i \in\{k, \ldots, n\}$ such that $x_{i}^{+}$has exactly $i-1$ zeros in $(0,1)$ and is positive near 0 , and $x_{i}^{-}$has exactly $i-1$ zeros in $(0,1)$ and is negative near 0 .

Theorem 23. Let (A1), (A2), (H1), and (H4) hold. Assume that the following condition holds for some $k, n \in \mathbb{N}$ with $k \leq n$ :

$$
r<\frac{\lambda_{k}}{f_{0}}<\frac{\lambda_{n}}{f_{0}} .
$$

Then problem (1) possesses $n-k+1$ pairs solutions $u_{i}^{+}$and $x_{i}^{-}$ for $i \in\{k, \ldots, n\}$ such that $x_{i}^{+}$has exactly $i-1$ zeros in $(0,1)$ and is positive near 0 , and $x_{i}^{-}$has exactly $i-1$ zeros in $(0,1)$ and is negative near 0 .

Theorem 24. Let (A1), (A2), (H1), and (H5) hold. Assume that the following condition holds for some $k, n \in \mathbb{N}$ with $k \leq n$ :

$$
\frac{\lambda_{k}}{f_{0}}<\frac{\lambda_{n}}{f_{0}}<r<+\infty .
$$

Then problem (1) possesses $n-k+1$ pairs solutions $x_{i}^{+}$and $x_{i}^{-}$ for $i \in\{k, \ldots, n\}$ such that $x_{i}^{+}$has exactly $i-1$ zeros in $(0,1)$ and is positive near 0 , and $x_{i}^{-}$has exactly $i-1$ zeros in $(0,1)$ and is negative near 0 .
Theorem 25. Let (A1), (A2), (H1), and (H6) hold. Assume that the following condition holds for some $k, n \in \mathbb{N}$ with $k \leq n$ :

$$
\frac{\lambda_{k}}{f_{\infty}}<\frac{\lambda_{n}}{f_{\infty}}<r<+\infty .
$$

Then problem (1) possesses $n-k+1$ pairs solutions $x_{i}^{+}$and $x_{i}^{-}$ for $i \in\{k, \ldots, n\}$ such that $x_{i}^{+}$has exactly $i-1$ zeros in $(0,1)$ and is positive near 0 , and $x_{i}^{-}$has exactly $i-1$ zeros in $(0,1)$ and is negative near 0 .

Remark 26. When $k=l=0$, the authors of [2-7] studied the existence of the solutions of the problem (1) by fixed point theory on cones, while we study the unilateral global bifurcation and the existence of nodal solutions of problem (1) for the cases of $f_{0}, f_{\infty} \in[0, \infty]$ by Dancer [33]. Thus, in this sense, our results partially extend and improve the corresponding results of [2-7].

Remark 27. The main methods used in this work are unilateral global bifurcation techniques and the approximation of connected components, which are different from the methods used in [13-15, 27-30, 34, 37]. Moreover, we consider the cases of $f_{0} \notin(0,+\infty)$ or $f_{\infty} \notin(0,+\infty)$, while the authors of $[13-15,27-30,34,37]$ only studied the cases of $f_{0}, f_{\infty} \in$ $(0, \infty)$.

Remark 28. Since Benedikt [18-22] has established the spectrum structure of the corresponding eigenvalue problems, in the following, one can study the existence of nodal solutions for the problems in Benedikt [18-22] by applying the bifurcation techniques in this paper.

Remark 29. When $k=l=0$, Korman [23] and Rynne [24] investigated the nodal properties of the solutions for problem (1) by applying the bifurcation techniques. Thus, in this sense, our results partially extend and improve the corresponding results of Korman [23] and Rynne [24].

Remark 30. We consider the cases of $f_{0}, f_{\infty} \notin(0, \infty)$ by Dancer [33], while the authors of [27] (when $k=0, l \neq 0$ ) only studied the cases of $f_{0}=\infty, f_{\infty} \in(0, \infty)$ by Rabinowitz [8]. Hence, Theorems 13-20 extend and improve Theorem 3.1 of [27] in some sense.

Remark 31. When $k=0, l=\beta(t)$, Ma et al. [28] only studied the cases of $f_{0}, f_{\infty} \in(0, \infty)$. Furthermore, one can study the cases of $f_{0}, f_{\infty} \notin(0, \infty)$ of [28] by using similar methods in this paper.

Remark 32. The authors of $[30,31]$ only studied the cases of $f_{0}, f_{\infty} \in(0, \infty)$ and $f_{0}=\infty, f_{\infty} \in(0, \infty)$ by Rabinowitz [8], respectively, while we consider the cases of $f_{0}, f_{\infty} \notin$ $(0, \infty)$ by Dancer [33]. Hence, Theorems 13-20 extend and improve the corresponding results of $[30,31]$ in some sense.

\section{Competing Interests}

The author declares that there is no conflict of interests regarding the publication of this paper. 


\section{Acknowledgments}

The author was supported by the NSFC (no. 11561038) and the National Science Foundation of Gansu (no. 145RJZA087).

\section{References}

[1] R. P. Agarwal and Y. M. Chow, "Iterative methods for a fourth order boundary value problem," Journal of Computational and Applied Mathematics, vol. 10, no. 2, pp. 203-217, 1984.

[2] R. Y. Ma and H. P. Wu, "Positive solutions of a fourth-order twopoint boundary value problem," Acta Mathematica Scientia, Series A, vol. 22, no. 2, pp. 244-249, 2002.

[3] R. Ma and C. C. Tisdell, "Positive solutions of singular sublinear fourth-order boundary value problems," Applicable Analysis, vol. 84, no. 12, pp. 1199-1220, 2005.

[4] Q. Yao, "Positive solutions for eigenvalue problems of fourthorder elastic beam equations," Applied Mathematics Letters, vol. 17, no. 2, pp. 237-243, 2004

[5] Q.-1. Yao, "Solvability of an elastic beam equation with Caratheodory function," Mathematica Applicata, vol. 17, no. 3, pp. 389-392, 2004.

[6] C. Zhai, R. Song, and Q. Han, "The existence and the uniqueness of symmetric positive solutions for a fourth-order boundary value problem," Computers \& Mathematics with Applications, vol. 62, no. 6, pp. 2639-2647, 2011.

[7] J. R. Webb, G. Infante, and D. Franco, "Positive solutions of nonlinear fourth-order boundary-value problems with local and non-local boundary conditions," Proceedings of the Royal Society of Edinburgh Section A: Mathematics, vol. 138, no. 2, pp. 427-446, 2008.

[8] P. H. Rabinowitz, "Some global results for nonlinear eigenvalue problems," Journal of Functional Analysis, vol. 7, no. 3, pp. 487513, 1971.

[9] P. H. Rabinowitz, "Some aspects of nonlinear eigenvalue problems," The Rocky Mountain Journal of Mathematics, vol. 3, pp. 161-202, 1973.

[10] C. P. Gupta and J. Mawhin, "Weighted eigenvalue, eigenfunctions and boundary value problems for fourth order ordinary differential equations," in Recent Trends in Differential Equations, vol. 1 of World Scientific Series in Applicable Analysis, pp. 253-267, World Scientific, 1992.

[11] A. C. Lazer and P. J. McKenna, "Global bifurcation and a theorem of Tarantello," Journal of Mathematical Analysis and Applications, vol. 181, no. 3, pp. 648-655, 1994.

[12] Y. Liu and D. O'Regan, "Bifurcation techniques for fourth order m-point boundary value problems," Dynamics of Continuous, Discrete and Impulsive Systems. Series A, Mathematical Analysis, vol. 18, no. 2, pp. 215-234, 2011.

[13] R. Ma, "Nodal solutions for a fourth-order two-point boundary value problem," Journal of Mathematical Analysis and Applications, vol. 314, no. 1, pp. 254-265, 2006.

[14] R. Ma, "Nodal solutions of boundary value problems of fourthorder ordinary differential equations," Journal of Mathematical Analysis and Applications, vol. 319, no. 2, pp. 424-434, 2006.

[15] R. Ma, C. Gao, and X. Han, "On linear and nonlinear fourthorder eigenvalue problems with indefinite weight," Nonlinear Analysis: Theory, Methods \& Applications, vol. 74, no. 18, pp. 6965-6969, 2011.

[16] B. P. Rynne, "Global bifurcation for $2 m$ th-order boundary value problems and infinitely many solutions of superlinear problems," Journal of Differential Equations, vol. 188, no. 2, pp. 461-472, 2003.

[17] A. Kratochvíl and J. Nečas, "On discreteness of spectrum of nonlinear Sturm.Liouville problem of the fourth order," Commentationes Mathematicae Universitatis Carolinae, vol. 12, no. 4, pp. 639-653, 1971 (Russian).

[18] J. Benedikt, "Uniqueness theorem for $p$-biharmonic equations," Electronic Journal of Differential Equations, vol. 53, pp. 1-17, 2002.

[19] J. Benedikt, "On simplicity of spectra of $p$-biharmonic equations," Nonlinear Analysis: Theory, Methods \& Applications, vol. 58, no. 7-8, pp. 835-853, 2004.

[20] J. Benedikt, "On the discreteness of the spectra of the Dirichlet and Neumann pbiharmonic problem," Abstract and Applied Analysis, no. 9, pp. 777-792, 2004.

[21] J. Benedikt, "Continuous dependence of eigenvalues of $p$ biharmonic problems on p," Communications on Pure and Applied Analysis, vol. 12, no. 3, pp. 1469-1486, 2013.

[22] J. Benedikt, "Ordinary p-biharmonic problems," Proceedings in Applied Mathematics and Mechanics, vol. 31, pp. 1-3, 2008.

[23] P. Korman, "Uniqueness and exact multiplicity of solutions for a class of fourth-order semilinear problems," Proceedings of the Royal Society of Edinburgh, Section A: Mathematics, vol. 134, no. 1, pp. 179-190, 2004.

[24] B. P. Rynne, "Infinitely many solutions of superlinear fourth order boundary value problems," Topological Methods in Nonlinear Analysis, vol. 19, no. 2, pp. 303-312, 2002.

[25] U. Elias, "Eigenvalue problems for the equations $L y+\lambda p(x) y=$ 0," Journal of Differential Equations, vol. 29, no. 1, pp. 28-57, 1978.

[26] U. Elias, Oscillation Theory of Two-Term Differential Equations, vol. 396 of Mathematics and Its Applications, Kluwer Academic Publishers, Dordrecht, The Netherlands, 1997.

[27] J. Xu and X. Han, "Nodal solutions for a class of fourthorder two-point boundary value problems," Boundary Value Problems, vol. 2010, Article ID 570932, 11 pages, 2010.

[28] R. Ma, H. Wang, and M. Elsanosi, "Spectrum of a linear fourthorder differential operator and its applications," Mathematische Nachrichten, vol. 286, no. 17-18, pp. 1805-1819, 2013.

[29] R. Ma and C. Gao, "Nodal solutions of a nonlinear eigenvalue problem of the Euler-Bernoulli equation," Journal of Mathematical Analysis and Applications, vol. 387, no. 2, pp. 1160-1166, 2012.

[30] W. Shen, "Existence of nodal solutions of a nonlinear fourthorder two-point boundary value problem," Boundary Value Problems, vol. 2012, article 31, 2012.

[31] W. Shen, "Global structure of nodal solutions for a fourth-order two-point boundary value problem," Applied Mathematics and Computation, vol. 219, no. 1, pp. 88-98, 2012.

[32] W. Shen and T. He, "Bifurcation from interval and positive solutions for a class of fourth-order two-point boundary value problem," Boundary Value Problems, vol. 2013, no. 1, article 170, pp. 1-12, 2013.

[33] E. N. Dancer, "On the structure of solutions of non-linear eigenvalue problems," Indiana University Mathematics Journal, vol. 23, pp. 1069-1076, 1974.

[34] G. Dai and R. Ma, "Unilateral global bifurcation phenomena and nodal solutions for p-Laplacian," Journal of Differential Equations, vol. 252, no. 3, pp. 2448-2468, 2012.

[35] G. Dai, "Bifurcation and nodal solutions for p-Laplacian problems with non-asymptotic nonlinearity at 0 or $\infty$," Applied Mathematics Letters, vol. 26, no. 1, pp. 46-50, 2013. 
[36] G. Dai, R. Ma, and H. Wang, "igenvalues, bifurcation and onesign solutions for the periodic p-laplacian," Communications on Pure and Applied Analysis, vol. 12, no. 6, pp. 2839-2872, 2013.

[37] G. Dai and X. Han, "Global bifurcation and nodal solutions for fourth-order problems with sign-changing weight," Applied Mathematics and Computation, vol. 219, no. 17, pp. 9399-9407, 2013.

[38] G. T. Whyburn, Topological Analysis, Princeton University Press, Princeton, NJ, USA, 1958.

[39] R. Ma and Y. An, "Global structure of positive solutions for nonlocal boundary value problems involving integral conditions," Nonlinear Analysis: Theory, Methods \& Applications, vol. 71, no. 10, pp. 4364-4376, 2009.

[40] G. Dai, R. Ma, and Y. Lu, "Bifurcation from infinity and nodal solutions of quasilinear problems without the signum condition," Journal of Mathematical Analysis and Applications, vol. 397, no. 1, pp. 119-123, 2013.

[41] P. H. Rabinowitz, “On bifurcation from infinity," Journal of Differential Equations, vol. 14, pp. 462-475, 1973.

[42] A. Ambrosetti, M. Calahorrano, and F. Dobarro, "Global branching for discontinuous problems," Commentationes Mathematicae Universitatis Carolinae, vol. 31, no. 2, pp. 213-222, 1990. 


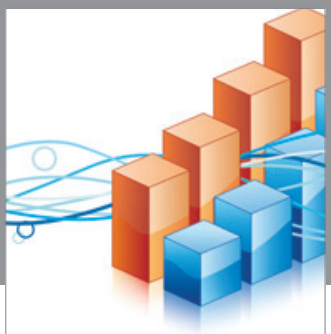

Advances in

Operations Research

vatem alat4

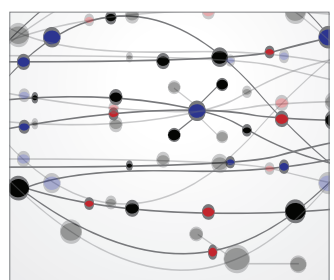

\section{The Scientific} World Journal
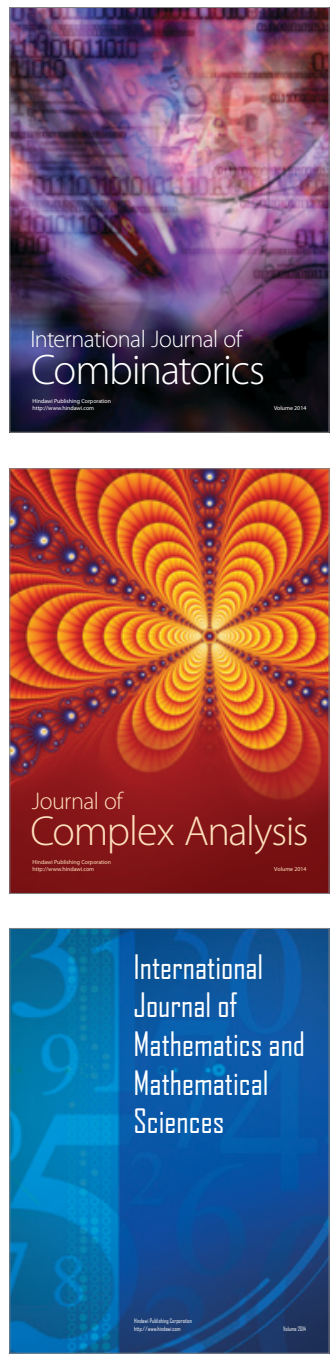
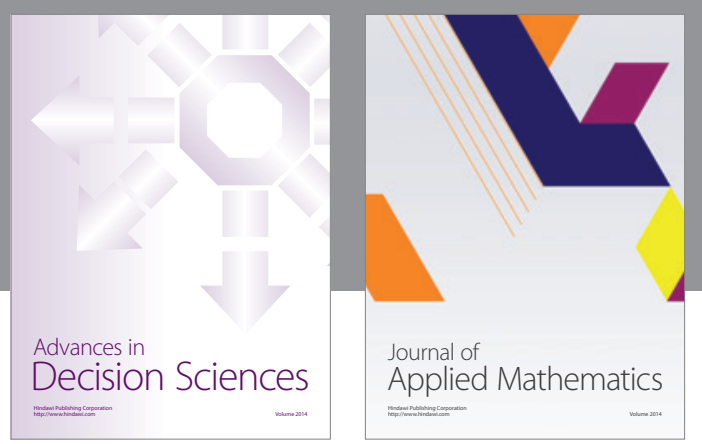

Algebra

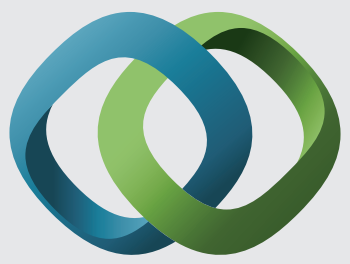

\section{Hindawi}

Submit your manuscripts at

http://www.hindawi.com
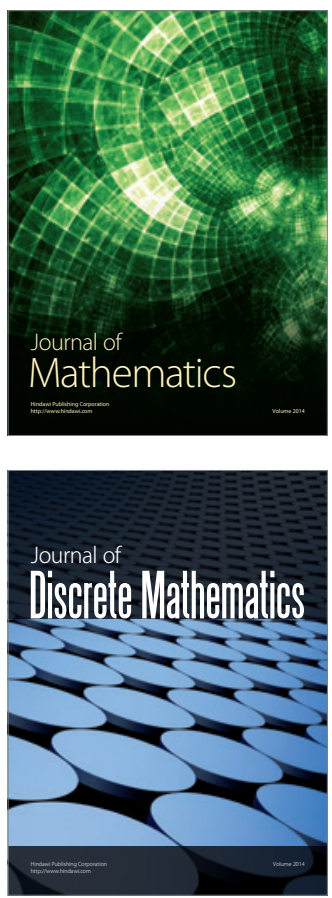

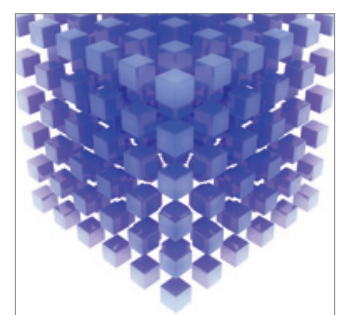

Mathematical Problems in Engineering
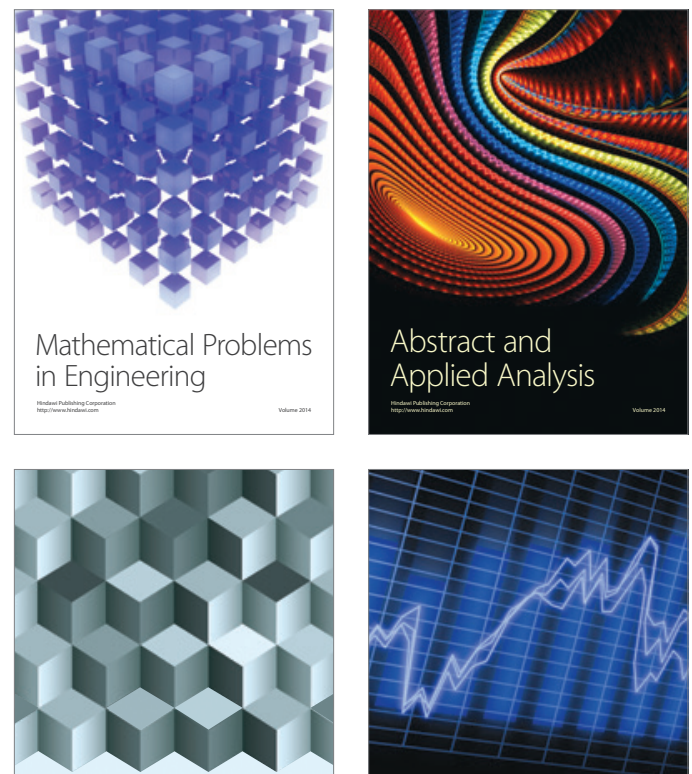

Journal of

Function Spaces

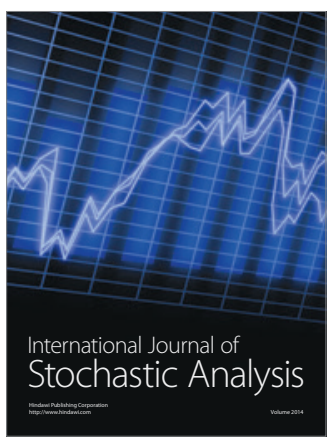

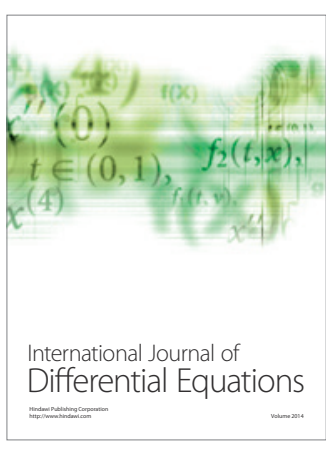
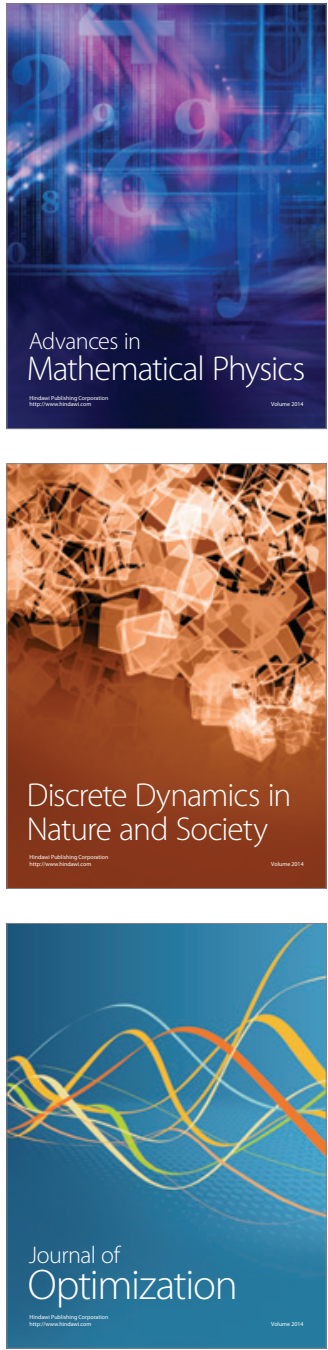